Article

\title{
Evaluating the Stressors Impacting Rescued Reptilian Wildlife
}

\author{
Harsh Kirpal Pahuja 1, Edward Narayan 1,* \\ 1 School of Agriculture and Food Sciences, Faculty of Science, University of Queensland, St Lucia, QLD 4072, \\ Australia \\ * Correspondence: e.narayan@uq.edu.au; Tel.: +61-7-5460-1693
}

\begin{abstract}
Simple Summary: Stress plays a vital role in influencing the survivability of rescued wildlife undergoing treatment and/or rehabilitation. In this study we identified 4 degrees of stressor categories (preliminary, primary, secondary and tertiary) in rescued reptiles admitted at the Adelaide Koala and Wildlife Hospital, South Australia. The study revealed that reptiles are highly susceptible to preliminary stressors in the face of urbanization, but majority of these reptiles are released back into wild. On the other hand, reptiles are particularly vulnerable to succumbing to death in the face of primary and secondary stressors. The study also revealed that reptiles are more likely to encounter stressors, and as a result be hospitalized, during the breeding season.
\end{abstract}

\begin{abstract}
Direct and indirect anthropogenic factors play a massive role in driving wildlife species towards extinction. Longitudinal retrospective studies identify key 'factors' responsible for the decline in numbers of wildlife, however, lack the reasoning behind the events leading to mortality. The overarching aim of this study was to categorize these 'factors' into different stressor categories faced by reptiles to understand its impact on an individual, and to compare how each stressor category influences the survival of an individual. The results from this study indicated that almost half of the number of reptiles being hospitalized were due to exposure to preliminary stressors such as lawn mowing incidents and pet attack. Primary and secondary admissions were fairly equal in number, however the mortality rate for secondary admissions was drastically high $(\sim 80 \%)$. The discussion integrates species' ecology and stress physiology which can prove to have multi-faceted benefits across the fields of ecology and animal welfare. Ecologists can use the results from this study to comprehend species' activity patterns to better plan reptilian conservation programs, whereas, for wildlife clinicians and rehabilitators, assignment of stressor categories could be a beneficial tool for bolstering the welfare monitoring program for small native reptiles in clinical settings.
\end{abstract}

Keywords: rehabilitation; stress; reptiles; injury; disease; euthanasia; trauma; clinical care

\section{Introduction}

The literature has substantive evidence to highlight the role of anthropogenic activities in negatively impacting wildlife populations, at a national scale [1-3], as well as globally [4-8]. The contribution of ever increasing urbanisation and population growth rates, in driving wildlife populations towards extinction is also well explored within the literature [9-11]. The activities associated with urbanisation and population growth result in habitat loss and fragmentation, thus, indirectly exposing wildlife to various biotic and abiotic environmental stressors [3,12]. Almost all retrospective studies identify both, direct and indirect extrinsic stressors that result in wildlife being admitted to wildlife clinics. A major limitation in these retrospective studies, however, is a lack of understanding of the impact of such environmental stressors on the physiology of an individual which might plausibly be an underlying cause of mortality in rescued wildlife. In order to understand the magnitude of impact of such stressors at an individual level, it is essential to delve deeper into the basic functioning of stress response.

Although the definition of stress is ambiguous, it is universally accepted that stressors are causative factors of stress, and when exposed to a stressor, the animal initiates a stress response in order to counteract the impact of stressor [13]. Contrary to the common notion, stress response is not always detrimental to an individual, but rather functions as 
a coping mechanism to deal with 'stressful' environments and to maximise its chances of survival in that situation [14]. The basic mechanism of a stress response is to modify an individual's physiology and to divert mobilized energy only to those systems that are vital for survival and to inhibit the non-essential body systems [15]. Despite the fact that stress response plays a vital role in daily activities such as foraging, breeding and so on [14,16]; when any population of wild animals is exposed to a stressor for a prolonged period of time (chronic stress) they respond to it either by adapting to the stressor by implementing behavioural and/or physiological plasticity or by altering their distribution range, for example, by migrating to a different non-stressful environment [17] as means of avoiding the stressor. Reptiles, however, have little to no capacity to migrate over long distances and are thus extremely vulnerable not only to direct anthropogenic stressors, but also to anthropogenic induced environmental stressors (AIES) [18]. Temperature dependent sex determination in reptiles aggravates the risk of AIES (e.g. climate change) resulting in skewed populations to an extent of complete demographic collapse [19]. The negative consequences of climate change are more pronounced in small lizard species because of their proficiency in maintaining near-constant body temperatures making them vulnerable to micro-thermal fluctuations [20]. Although this implies that analysis of existing reptile rescue databases could provide valuable insights into biodiversity health and illuminate the complexity of microclimates occupied by reptilian populations, retrospective studies of rescued reptiles are rare [21].

Wildlife rescue databases serve as an important tool in the field of ecological research to explain the negative impacts of anthropogenic activities on wildlife and to improve urban planning by taking appropriate measures to ameliorate the negative consequences of such activities [22]. Furthermore, categorizing direct and indirect anthropomorphic perturbations based on stressor categories at an individual level can help in understanding the synergistic impact of stressors on an individual and thereby assist in minimising the intensity of stressors during rescue; treatment; throughout rehabilitation and during release [12]. For example, a lizard kept as an illegal pet might seem physically healthy and ready for release in the wild, but may be exposed to preliminary stressor in the form of inappropriate housing conditions as a result of which it might exhibit a primary stress response in the form of temporary abnormal behaviour.

The primary aim of this study was to categorize anthropomorphic and anthropogenic induced environmental perturbations based on stressor categories, to understand the complex notion of stress and comprehend how we can ameliorate the impact of such stressors. We then compared and contrasted the impact of different stressor categories on clinical outcome of rescued reptiles. This also allowed us to test the conceptual stress physiology models since we predicted that reptiles that are under the influence of secondary and tertiary stressors would more likely be higher up on the reactive homeostasis range (see Romero et al. [23]) resulting in higher mortality given the prolonged exposure to stress and higher magnitude of secondary and tertiary stressors as compared to individuals that are under the influence of preliminary and primary stressors. We also predicted that, since reptiles generally are generally active during the summers [24-29], there will be a significant increase in number of rescues during the warmer months of the year. The results from this retrospective analysis will allow us to integrate distinct sciences of stress physiology and conservation biology to better design and implement reptile conservation programmes by improving urban planning. In addition, a standardized categorisation of stressors may have potential ramifications in enhancing clinical care of injured wildlife in the future.

\section{Materials and Methods}

\subsection{Study site-}

The clinical data of rescued wildlife was obtained from Adelaide Koala and Wildlife Hospital (AKWH) (3496’39.2" S, $138^{\circ} 5^{\prime} 35.0^{\prime \prime}$ E). AKWH is located in Plympton which is the inner urban part of the city capital, Adelaide. AKWH is a non-profit organisation inaugurated in February 2014 that relies solely on magnanimous financial support and in-kind 
donations from generous samaritans. The staff and volunteers at AKWH provide free emergency services for injured wildlife and predominantly work towards rescuing and releasing, treated and rehabilitated wildlife. In addition, the hospital also has species-specific enriched enclosures for permanent residency of wildlife that cannot be released back into the wild. In addition to veterinary care and rehabilitation, AKWH also participates in educational outreach programs and promotes research and development by collaborating with various sectors. As this is a desktop only study animal ethics was not required for this project.

\subsection{Data collection-}

The clinical database of wildlife pertaining to all rescued admissions between $1^{\text {st }}$ January 2016 to $31^{\text {st }}$ December 2020 was obtained from AKWH. The database was then restructured to include clinical data for all the reptiles rescued throughout the aforementioned period of interest of study. The data was then systematically organized into Microsoft Excel with additional information such as species (shingleback lizard, bearded dragon, short-necked turtle, etc.); age (hatchling, juvenile and adult); gender (male and female); rescue location; cause of admission and outcome (died, euthanized, released in care, released in wild). The rescue location was segregated into rural, urban and suburban categories (refer section 2.4). The causes of admission were used to classify stressor categories (refer section 2.5)

\subsection{Study species-}

\subsubsection{Blue tongue lizard}

Blue tongue lizards belong to the genus Tiliqua of the Scinicidae family and comprises of eight species, of which only eastern/common blue tongue lizard (T. scincoides), pygmy blue tongue lizard (T. adelaidensis) and shingleback lizard (T. rugosa) are found in the range of wildlife rescues undertaken by AKWH [25,30]. Pygmy blue tongue lizard is an 'Endangered' species [25] because its population is only restricted to remnant private grasslands in the mid-north of South Australia [31] as a result of which it is rarely rescued, if ever. On the other hand the common blue tongue lizard is considered as 'Least Concern' as per the IUCN [30] because of its widespread distribution. This species (T. scincoides) breeds during the warmers months of the year (generally during the summers, between December and February) and gives birth to a large number of precocial young ones [3,32].

\subsubsection{Shingleback lizard}

Tiliqua rugosa commonly known as the shingleback lizard, also belongs to the family of skinks- Scinicidae, and shares a common genus with the blue tongue lizard. This species is widespread throughout its range and is classified as 'Least Concern' as per the IUCN [33]. These lizards have a short breeding season and breed only during the months of September and November. Unlike most lizards, this species presents an unusual monogamous breeding habit and are viviparous, giving birth to 1-4 relatively large offspring [34].

\subsubsection{Bearded dragon}

Bearded dragon belongs to genus Pogona of the Agamidae family and comprises of six species, of which only central ( $P$. vitticeps) and eastern bearded dragon ( $P$. barbata) are found in the range of wildlife rescues undertaken by AKWH $[26,27]$. Both species are oviparous, having a long breeding season throughout the warmer months of the year, and are classified as 'Least Concern' as per the IUCN [26,27].

\subsubsection{Short-necked turtle}

Short-necked turtle scientifically named as Emydura macquarii macquarii is a subspecies of the more commonly found Southern river turtle (Emydura macquarii) [29]. E. macquarii macquarii is commonly found in the Macquarie river of South Australia and the breeding season varies from mid-September-December up to January. The females are oviparous and lay 1-3 clutches every year of varying sizes [29].

\subsubsection{Long-necked turtle}


Chelodinia longicollis commonly known as eastern long neck turtle is widespread across a variety of water bodies ranging from freshwater rivers to ephemeral water holes throughout South East Australia [28]. The species is not listed in the IUCN; however, it is classified by [28] as 'Least Concern'. This species has a short breeding season lasting throughout September and the female lays 6-25 eggs during the nesting period between October to January [28].

\subsection{Categorising location-}

The datasheet for each individual included the location from which it was rescued and was included in the database provided. On the basis of information provided by Australia Bureau of Statistics and method outlined in [12,35], the rescue location was used to classify all rescued individuals in four habitat categories, namely- rural, urban, suburban and unknown. We used the Google Earth program to input rescue location to obtain a satellite view for categorising the individuals. An individual was categorised under rural habitat if it was rescued from a low population density area surrounded mainly by trees and located outside towns and cities. An individual rescued from a high population density area surrounded mainly by buildings and industries was categorised under urban habitat. Sub-urban habitat category included individuals rescued from areas that were more densely populated than rural areas but less densely populated than urban areas. Individual data sheets that did not specify any rescue location were classified under the unknown category.

\subsection{Categorising stressors-}

The database of rescued wildlife included the cause of presenting the animal to the clinic and the clinical diagnosis for each individual. As inferred from Schreck et al. [36] and Janssen et al. [12], these records were classified into four stressor categories (resulting in respective stress levels) as preliminary stressors (P1S), primary stressors (P2S), secondary stressors (SS) and tertiary stressors (TS). P1S included any external stimulus that had the potential and/or led to any form of physical (including but not limited to vehicle collisions and animal induced trauma), physiological (including but not limited to feed and water deprivation and extreme temperatures) and/or psychological stress (including but not limited to confinement and/inappropriate environments and surroundings) resulting in admission of the individual to the hospital [12,36]. P2S included any external stimulus that induced, or were potentially capable of inducing a primary stress response causing an increase in catecholamine and steroid hormones (including but not limited to complete/partial immobility, temporary abnormal behaviour, bleeding, laceration and abrasion) [12,36,37]. Similarly, SS included any external stimulus that induced a secondary stress response resulting in alterations at a cellular (changes in heat shock/stress protein production); metabolic (changes in glucose and lactate production), osmoregulatory (changes in osmolality of specific ions), haematological (changes in leukocytes and haemoglobin levels) and immune function (changes in antibody production). Examples include, but not limited to fracture, disease and haemorrhage [12,36,37]. TS included any external stimulus that affected the growth, behaviour, fitness and survival of an individual (including but not limited to permanent abnormal behaviour, permanent body disfigurement, permanent suppression of neurological responses and/or other vital senses) [12,36]. Although traditional classification of stressors is of theoretical significance and classifies stressors on a broader scale (see Asterita [38]), it cannot decipher the complex notion of 'stress' and therefore, a more practical classification of stressor categories is essential to better understand the impact of stressors at an individual level (Figure 1) which can then be extrapolated to a population level for better urban planning which is vital to reptile conservation and management. 


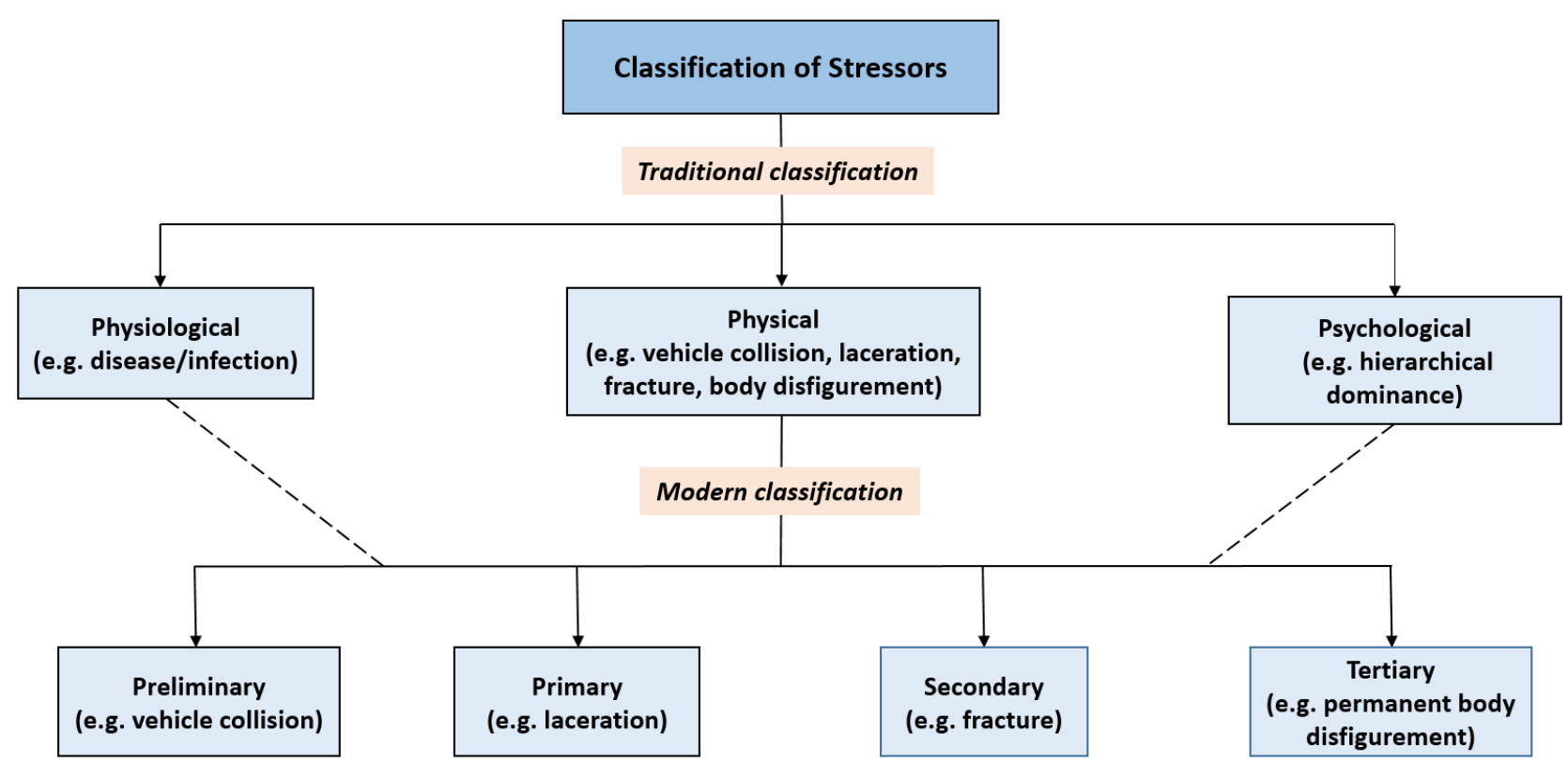

Figure 1. A diagram illustrating traditional broad classification of stressors into three categories which can be further categorized into a more practical modern classification. For example, as per the traditional classification- vehicle collision, laceration, fracture and body disfigurement would all be classified as a physical stressor however modern classification allows to further categorize these physical stressors to understand the impact of stressors (see chapter 2 classification). Dotted segments indicate that similar modern categorization of physiological and psychological can be achieved

\subsection{Statistical analysis-}

The database of rescued reptiles between 2016 and 2020 was over represented by the blue tongue lizard making the database largely unstable to carry out statistical analysis. In order to deal with this problem, we performed descriptive statistical analysis using Microsoft Excel to present general trends with respect to number of rescues per year; species' admission; age category; sex; rescue location; clinical outcome and stressor categories. We then compared the clinical outcome of all reptiles with each stressor category to identify which stressor category was more likely to result in mortality (including natural death in care or euthanasia based on welfare terms). In addition, since the sample size of blue tongue lizards was sufficient to perform statistical analysis, we preformed t-test assuming equal variances and we determined the level of significant difference was determined by $p$-value $<0.05$, to identify if there were any seasonal trends in number of rescues of blue tongue lizards. We assumed equal variance between breeding and non-breeding seasons' sample sizes because, rescuers attend every case they receive, irrespective of the potential effect of season. This rules out the chance of prejudice/bias between admissions thereby making it safe to assume that variance would be negligible.

\section{Results}

\subsection{General trends}

General trends refer to the variation in terms of number of rescues per year; species' rescued; location of rescue; age and sex category of rescued individuals and clinical outcome of rescued individuals. A summary of general trends for all rescued reptiles between 2016 and 2020 is represented in Table 1.

\subsubsection{Trends in number of rescues over the study period}

A total of 221 reptiles pertaining to five different species were rescued throughout the duration of the study period between 2016-2020. The year 2020 accounted for highest number of rescues accounting for $n=62$ during the study period in spite of the lockdown restrictions due to COVID-19. This was followed by the year 2018 accounting for $n=54$ rescues followed by $n=48$ in 2019; n=39 in 2017 and $n=18$ in 2016. 


\subsubsection{Trends in species admitted to the clinic}

Blue tongue lizards were rescued more than any other reptile over all 5 years and represented the majority of rescues at $73.3 \%(n=162)$ followed by long necked turtles at $7.6 \%(n=17)$ and shingleback lizards at $6.7 \%(n=15)$. Total unknown species pertained to $6.3 \%(n=15)$ including unknown lizards $(n=1)$ and unknown turtles ( $n=14)$. This was closely followed by bearded dragon at $4.9 \%(n=11)$ and only a single rescue of the short necked turtle $(n=1)$.

\subsubsection{Trends in age and sex category}

For all reptiles, age was categorised based on the life history stage, namely, hatchling, juvenile and adult. Majority of the reptiles rescued were adults $(43.4 \%, n=96)$ over all years and across all species that were rescued. This was followed by hatchlings $(10.8 \%, n=24)$ and juveniles $(9 \%, n=20)$. Note that $36.6 \%(n=81)$ of admissions were missing information on age category. In case of sex identification, only $12.6 \%$ of rescued reptiles could be successfully identified pertaining to $9 \%$ individual males $(n=20)$ and $3.6 \%$ individual females $(n=8)$. The remaining $87.3 \%(n=193)$ of individuals were noted as unidentified.

\subsubsection{Trends in location from which the animals were rescued}

Of total 221 rescued reptiles, 130 were from urban location, 10 from rural, 27 from sub-urban and 54 were unknown location.

\subsubsection{Trends in clinical outcome}

A positive caveat to note is that $64.2 \%(n=142)$ of all rescued reptiles were released which includes the categories noted as 'released into wild' $(n=114)$ and 'released into care' $(n=28)$. Total death after being rescued pertained to $31.6 \%$ $(n=70)$ which includes categories noted as 'euthanized' $(n=53)$ and 'died' $(n=17)$. Note that $4 \%(n=9)$ of admissions were missing information on clinical outcome of rescued reptiles. 
Table 1. A summary table illustrating all general trends (age, sex, location, clinical outcome, species' and year) in all reptile admissions at AKWH throughout the duration of 1 the study.

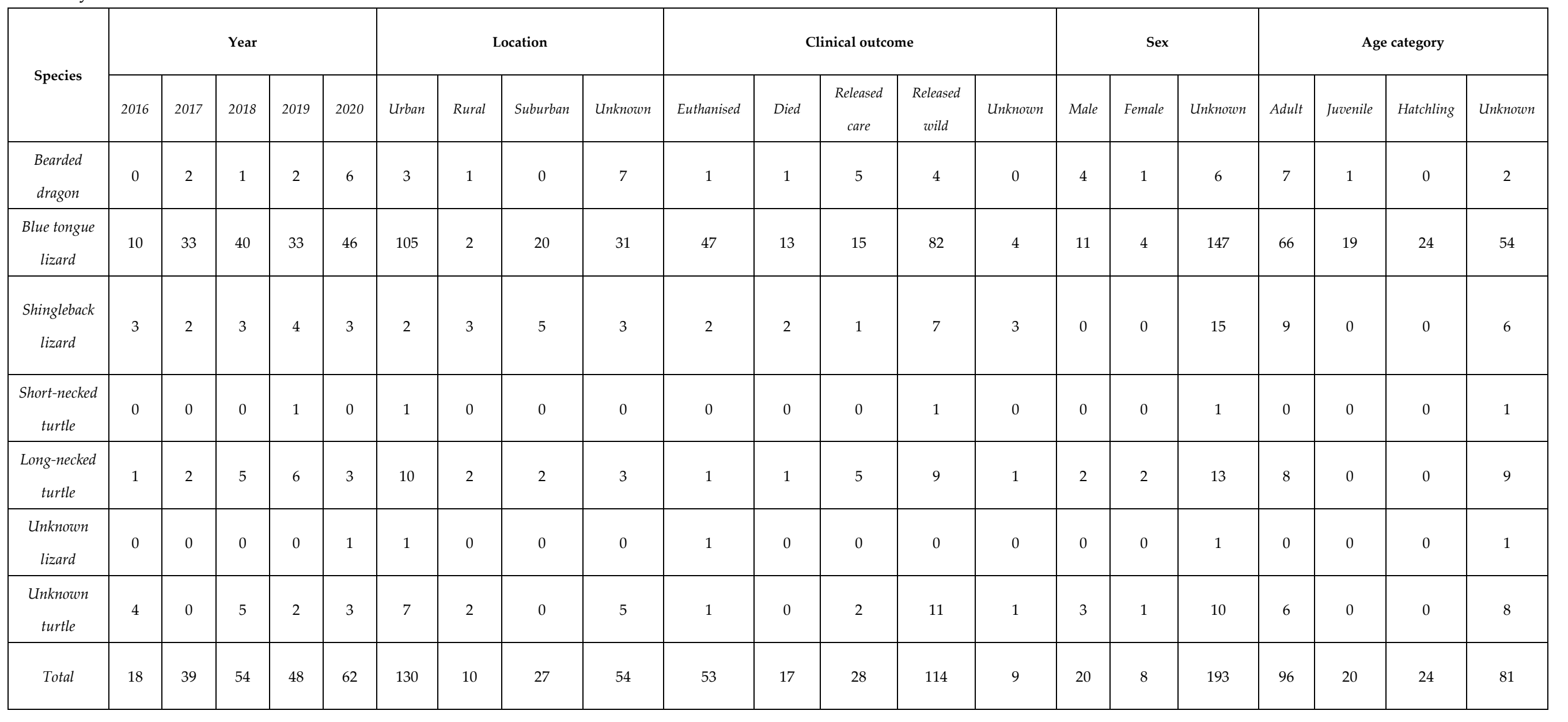




\subsection{Stressor categories}

Cause of rescue and clinical diagnosis was used to categorise stressors for each individual. The results from this study indicate that across all 5 species, most number of individuals were rescued for preliminary reasons accounting for 110 of total 221 rescues. The most common preliminary stressors for reptiles included pet attack, vehicle collision and machine injury from lawn mowing equipment. Other preliminary stressors such as surrendered pet, displaced and an unusual incidence of duct tape on face was also noted. Note that individuals were classified under preliminary stressor category when they were presented/diagnosed with reasons that fit under preliminary stressor category only (as mentioned above) with no information fitting in remaining three categories of stressors (primary, secondary and tertiary). After preliminary category, reptiles were commonly rescued for secondary stressors, accounting for 57 of total 221 cases. Secondary stressors such as fractured bones, puncture wounds, injury to vital organs and infection/diseases were identified. Note that individuals were classified under secondary stressor category when they were presented/diagnosed with reasons that fit under secondary stressor category only (as mentioned above) with no information fitting in remaining three categories of stressors (preliminary, primary and tertiary). Secondary stressor category was closely followed by primary stressors which accounted for 51 of total 221 admissions to the clinic. Primary stressors such as minor injuries, abrasions, wounds and lesions were noted and were all pooled under 'injury'. Other primary stressor such as 'trauma' and being 'flat' was also noted for some individuals. Note that individuals were classified under primary stressor category when they were presented/diagnosed with reasons that fit under primary stressor category only (as mentioned above) with no information fitting in remaining three categories of stressors (preliminary, secondary and tertiary). Tertiary stressor category was the least common cause of rescue in reptiles accounting for only 3 of total 221 cases. Tertiary stressor category such as blind individuals and individuals with permanent neurological disorder were noted. Note that individuals were classified under tertiary stressor category when they were presented/diagnosed with reasons that fit under tertiary stressor category only (as mentioned above) with no information fitting in remaining three categories of stressors (preliminary, primary and secondary). Categorisation of stressors for all species is summarised in Figure 2.

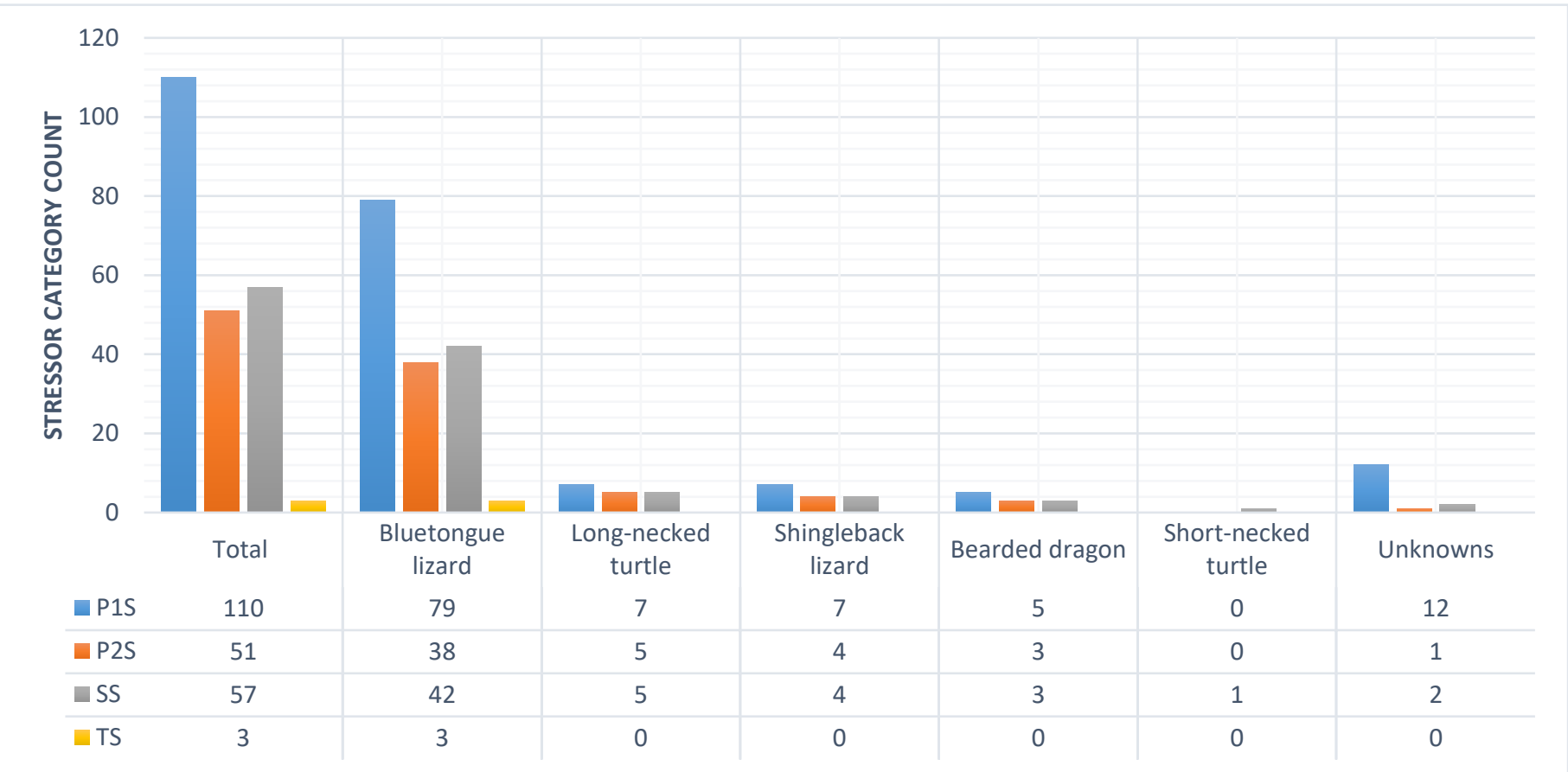

Figure 2. A graphical representation of different categories of stressors experience by different reptilian species admitted at AKWH between 2016 and 2020. The category 'Total' refers to the summation of all species and unknown reptilian species. The category 'Unknown' refers to admissions that were included under reptiles but could not be identified at a species level. 


\subsection{Comparing stressor categories with clinical outcome}

The results comparing stressor categories to clinical outcome of rescued reptiles (illustrated in Figure 3) indicate that, for reptiles admitted under preliminary stressor category only $(n=110), 10 \%(n=11)$ of admitted cases succumbed to death (including natural death in care or euthanasia based on welfare terms), whereas, in case of animals admitted for primary stressor category only $(n=51)$, mortality rate increases to more than double than that in case of preliminary category accounting for $25.5 \%$ ( $n=13$ of total 51 primary cases) of deceased reptiles. In the event of reptiles admitted for secondary stressor category, the mortality rate indicated an 8-fold increase compared to preliminary stressor category and more than 3-fold increase compared to the primary stressor category, accounting for a total of $78.9 \%$ ( $\mathrm{n}=45$ of total 57 secondary cases) of deceased cases. Conversely, it is also important to note that, as we move from preliminary to primary to secondary stressor categories a decrease in number of released reptiles (both in wild and care) was observed with the release rate dropping from $81.8 \%$ in case of preliminary category to $21 \%$ in case of secondary stressor category. Reptiles identified with tertiary stressor category were negligible $(n=3)$ and were thus considered insignificant to understand trends in this study ( $\mathrm{n}=2$ released in care/wild; $\mathrm{n}=1$ euthanized). Note that some individuals were missing information $(n=9)$ about their clinical outcome and were thus omitted from the figure.

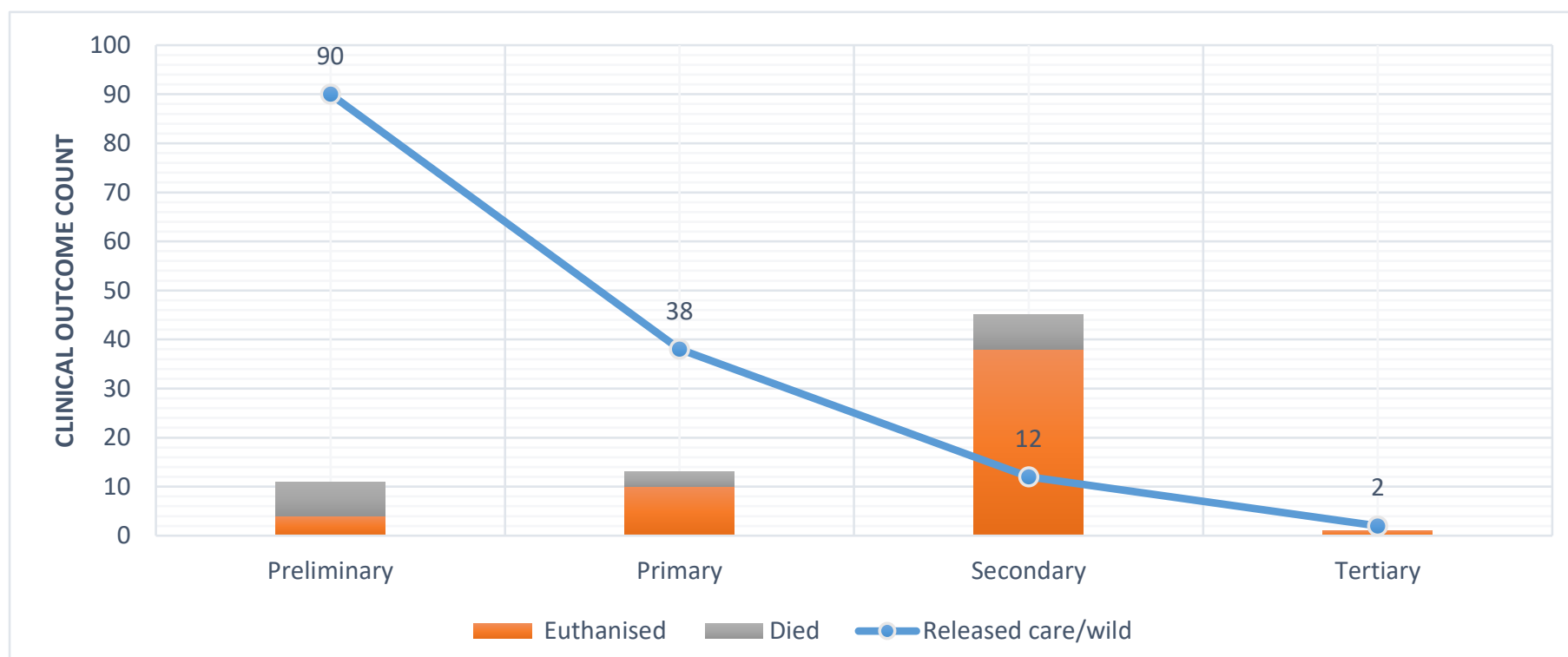

Figure 3. A graphical illustration of clinical outcome of reptiles with respect to different stressor categories experienced by reptiles admitted at AKWH between 2016 and 2020. Note that data labels indicate numbers for 'Released care/wild' category only. 


\subsection{Trends in monthly rescues for blue tongue lizards}

We observed an apparent seasonal trends in number of admissions of blue tongue lizards with higher number of rescues between early spring and early autumn (i.e. during relatively warmer months of the year) and lesser number of admissions during the rest of the year (i.e. during relatively colder months of the year). Statistical $t$-test assuming equal variance with a degree of freedom, $\mathrm{df}=10$ produced a $\mathrm{t}$-value of 4.194 and indicated that there was a significant increase $(p<0.001)$ in number of admissions of blue tongue lizards during the breeding season as compared to the non-breeding season (Figure 4) (refer discussion to access an in-depth review of life cycle of blue tongue lizard)

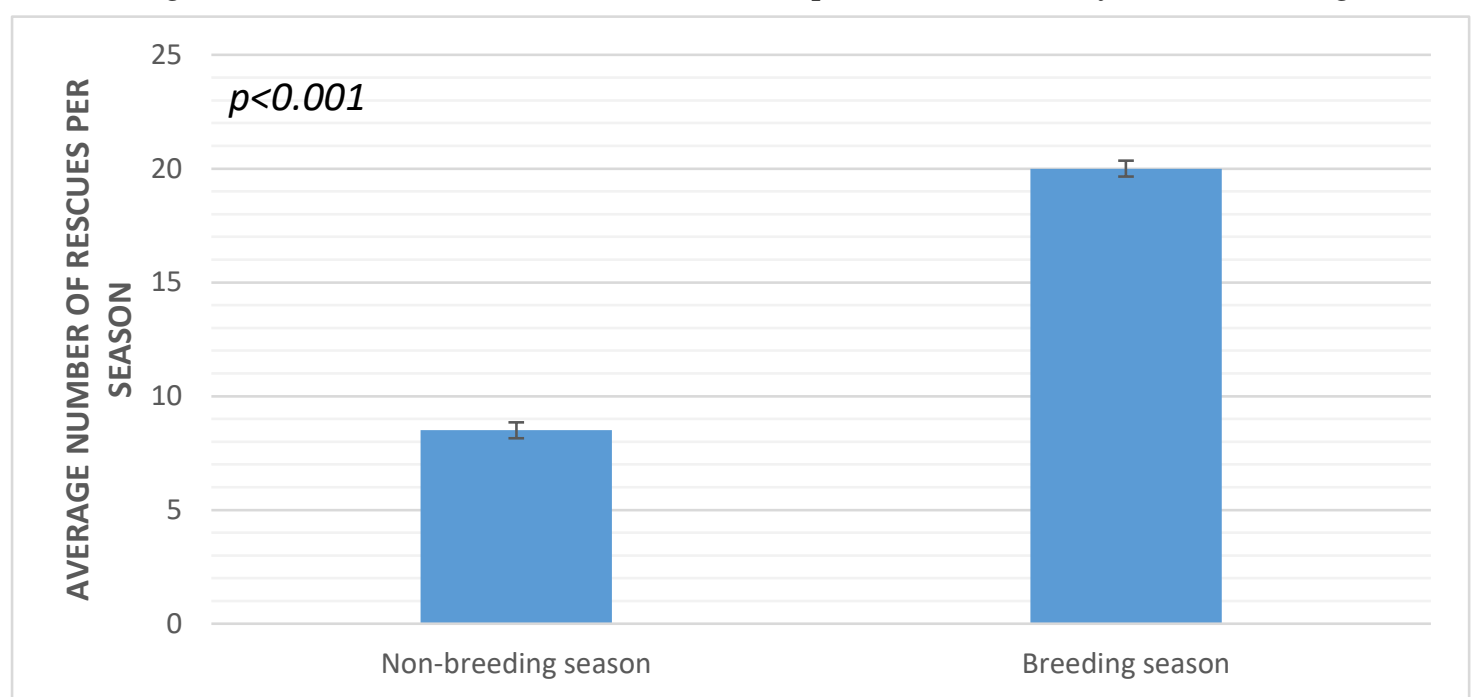

Figure 4. A graphical illustration depicting a significant increase in number of rescues during the breeding season. Note that the life-stages are broadly divided into breeding and non-breeding season based on blue-tongue life cycle. In reality the life-stages are much more complex involving stages such as pre-breeding, nesting period and so on.

\section{Discussion}

Wildlife rescue databases not only serve as a tool to indicate biodiversity and ecosystem health [4,22], but also serve as a repository to indicate specific encounters with an individual at a certain location representing certain age and sex [39]. This vital information can be used to gain an in-depth understanding of its biology [3,40] and to plan relevant conservation actions $[2,41]$. Sustained urban development and habitat fragmentation has resulted in shrinking of wildlife zones, and has forced human and reptile cohabitation which has inevitably led to an increase in the frequency of human-reptile conflict situations [22]. Several studies in the past have retrospectively analysed the causes of morbidity and mortality in rescued wildlife, however very few studies [12] have tried to understand the impact of those 'causes' on an individual, which is a plausible underlying cause of mortality in wildlife. The results from this study indicated that over the years more and more number of reptiles are being rescued, with the year 2020 accounting for highest number of rescues in spite of the COVID-19 restrictions. This result can be attributed to the restrictions imposed due to the pandemic resulting in citizens being confined to their homes. Since majority of these reptiles were 'urban-wild' (i.e. commonly found in and around human settlements) [42], the encounters between human-reptiles increased which could plausibly be the reason for more number of animals being reported, and thus being admitted at the wildlife centre. Nonetheless, irrespective of the pandemic, a consistent increase in number of rescues over the years, has been observed in almost all longitudinal studies conducting a retrospective analysis of injured wildlife admissions $[2,12,41]$. Blue tongue lizard was the most commonly rescued species which is consistent with several other studies [22,40] conducted in Australia. Hamer and McDonnell [42] explain that blue tongue lizards are commonly found in human dominated landscapes (such as backyards), resulting in frequent encounters with direct anthropogenic factors (such as pets and/or lawn mowing equipment). This explanation is also reinforced by our results, since a large proportion of these reptiles were in fact rescued from urban locations. 
A vast majority of reptiles were rescued for preliminary reasons such as minor idiopathic trauma, speculative pet attack with no major injuries, displaced individuals and surrendered pets, which resulted in majority of these individuals being released after superficial treatment. Admitting and releasing a huge number of preliminary cases could however negatively impact the functioning of the clinic by diverting resources from individuals requiring intensive care. The release rate of rescued reptiles dropped drastically as we moved from preliminary to primary to secondary stressor categories and conversely, as predicted, the mortality rate increased consistently as we moved from preliminary to primary to secondary stressor categories. A drastic increase in mortality rate for individuals admitted for secondary reasons, can be attributed to greater magnitude/intensity of the stressor and the duration for which the individual was under stress. Janssen et al. [12] explain that stressor magnitude and frequency are important underpinning factors for the recovery of rescued wildlife. In addition, the duration for which an individual is under stress is also a vital aspect differentiating acute and chronic stress [23,43]. Individuals experiencing secondary stress are likely to have experienced preliminary and primary prior to reaching secondary levels of stress, thereby increasing the total duration for which those individuals were under stress. Moreover, these individuals are also faced with the cumulative magnitude of preliminary, primary and secondary stressors resulting in higher mortality rates of individuals admitted for secondary stressors. Tertiary stressor category represented only 3 admissions and was thus considered insignificant to explain trends, however, since tertiary stressors refer to effects at a whole-individual level (including cases such as permanent body disfigurement, suppression of senses and so on) [12,36,37], we believe that the rate of 'release in care' would be higher as compared to 'mortality' and 'release in wild'.

As hypothesised, a significant increase in number of rescues of blue-tongue lizards was observed during the breeding season relative to the non-breeding season. Since the sample size of other rescued reptiles was not sufficient we could not identify seasonal trends for these reptiles. In case of blue tongue lizards, a consistent drop in number of rescues was observed starting from March until August, with the numbers starting to rise again from September. These results can be attributed to the life cycle of blue tongue lizard (Figure 5). During the colder months of the year, specifically during late autumn, winter and early spring (which is also the non-breeding season) this species undergoes hibernation due to which the number of rescues is low [3,40]. During the rest of the spring and throughout summer this species is active [24]. Males pursue females during spring which is the mating season for this species. After mating, the females give birth to precocial young during the 'nesting period', usually during summer between December and February [3,32]. A spike in number of rescues during the breeding season is in consistency with a study conducted by Koenig et al. [3] focusing on blue tongue lizards rescued in Sydney. We believe that a small rise in admissions during the month of March (see Figure 5) can be attributed to the dispersal of blue tongue lizards' hatchlings, since- (a) it is consistent with the gestation period and the approximate time of parturition in female blue tongue lizards [44] and (b) because of the precocial nature of the hatchlings of this species [32]. Future studies can focus on identifying whether the rescues conducted during 'hatching' season were significantly attributed to juveniles and hatchlings or not. Such casuistry details can help better plan conservation actions for seasonal species like the blue tongue. 


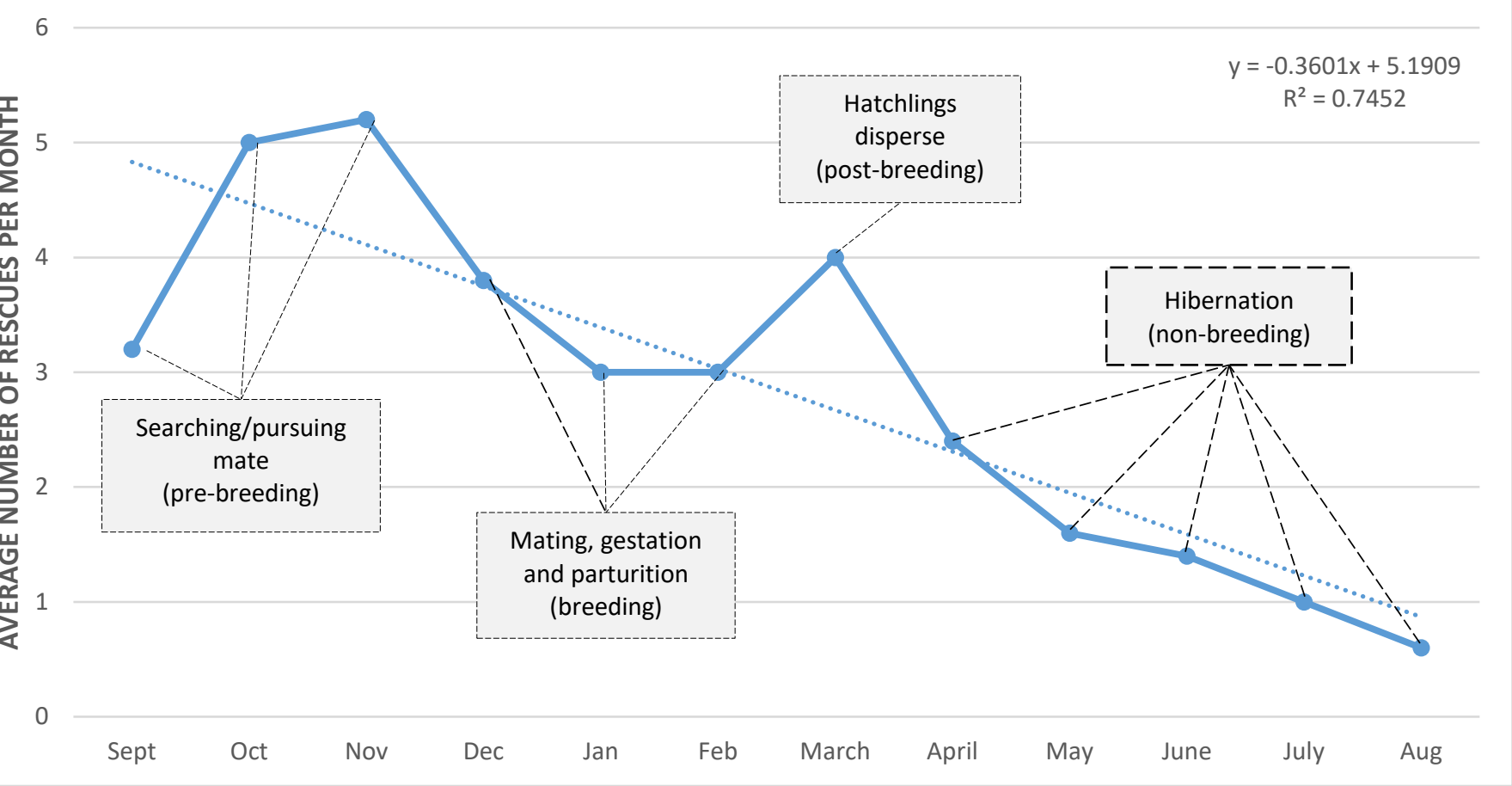

Figure 5. A graphical illustration of average number of admissions of blue tongue lizards at AKWH per month for the years 2016 to 2020. Note that trend line is imposed to emphasize the decrease in number of admissions during the late autumn and winter season.

\section{Conclusions}

The categories of stressors identified in this study could help wildlife clinicians and rehabilitators in trying to minimise the intensity and frequency of a stressor and thus the total duration for which an individual is exposed to stress. Furthermore, it could also help environmental biologists and ecologists to incorporate the role of species' biology and life cycle to better plan, organise and manage conservation based programmes by taking into account the major causative factors of mortality in reptiles. For individuals released after being treated for primary and secondary stressors, future studies should focus on tracking the survivability of these individuals after being released since it can help to allocate resources effectively. A large proportion of the database for rescued reptiles was missing information on animal specific characteristics such as sex and age which could help in excavating interesting findings related to an animal's biology.

Supplementary Materials: All primary data is available within the manuscript.

Author Contributions: “Conceptualization, E.N. and H.P.; methodology, E.N. and H.P.; formal analysis, E.N. and H.P.; investigation, E.N. and H.P; resources, E.N.; data curation, E.N. and H.P.; writing-original draft preparation, H.P.; writing-review and editing, E.N. and H.P; supervision, E.N.; project administration, E.N. All authors have read and agreed to the published version of the manuscript.

Funding: This research received no external funding.

Institutional Review Board Statement: Not applicable because this is a desktop based data study.

Data Availability Statement: The AKWH can be contacted to request original database.

Acknowledgments: We wish to express our appreciation to the entire team of Adelaide Koala and Wildlife Hospital. A special thanks to Dr. Phil Hutt and Joanne Sloan for consistently updating the database which formed the core of this project.

Conflicts of Interest: The authors declare no conflicts of interest. 


\section{References}

1. Scheelings, T.F.; Frith, S.E. Anthropogenic Factors Are the Major Cause of Hospital Admission of a Threatened Species, the Grey-Headed Flying Fox (Pteropus poliocephalus), in Victoria, Australia. PloS one 2015, 10, e0133638.

2. Taylor-Brown, A.; Booth, R.; Gillett, A.; Mealy, E.; Ogbourne, S.M.; Polkinghorne, A.; Conroy, G.C. The impact of human activities on Australian wildlife. PloS one 2019, 14, e0206958.

3. Koenig, J.; Shine, R.; Shea, G. The dangers of life in the city: patterns of activity, injury and mortality in suburban lizards (Tiliqua scincoides). Journal of Herpetology 2002, 62-68.

4. Schenk, A.N.; Souza, M.J. Major anthropogenic causes for and outcomes of wild animal presentation to a wildlife clinic in East Tennessee, USA, 2000-2011. PLoS One 2014, 9, e93517.

5. Brown, J.D.; Sleeman, J.M. Morbidity and mortality of reptiles admitted to the Wildlife Center of Virginia, 1991 to 2000. Journal of wildlife diseases 2002, 38, 699-705.

6. Molina-López, R.A.; Casal, J.; Darwich, L. Causes of morbidity in wild raptor populations admitted at a wildlife rehabilitation centre in Spain from 1995-2007: a long term retrospective study. PLoS One 2011, 6, e24603.

7. Romero, F.; Espinoza, A.; Sallaberry-Pincheira, N.; Napolitano, C. A five-year retrospective study on patterns of casuistry and insights on the current status of wildlife rescue and rehabilitation centers in Chile. Revista chilena de historia natural 2019, 92.

8. Orós, J.; Montesdeoca, N.; Camacho, M.; Arencibia, A.; Calabuig, P. Causes of stranding and mortality, and final disposition of loggerhead sea turtles (Caretta caretta) admitted to a wildlife rehabilitation center in Gran Canaria Island, Spain (19982014): a long-term retrospective study. PLoS One 2016, 11, e0149398.

9. Ceballos, G.; Ehrlich, P.R.; Barnosky, A.D.; García, A.; Pringle, R.M.; Palmer, T.M. Accelerated modern human-induced species losses: Entering the sixth mass extinction. Science advances 2015, 1, e1400253.

10. Ceballos, G.; García, A.; Ehrlich, P.R. The sixth extinction crisis: Loss of animal populations and species. Journal of Cosmology 2010, 8, 31 .

11. Barnosky, A.D.; Matzke, N.; Tomiya, S.; Wogan, G.O.; Swartz, B.; Quental, T.B.; Marshall, C.; McGuire, J.L.; Lindsey, E.L.; Maguire, K.C. Has the Earth's sixth mass extinction already arrived? Nature 2011, 471, 51-57.

12. Janssen, K.; Marsland, C.; Barreto, M.O.; Charalambous, R.; Narayan, E. Identifying the stressors impacting rescued avian wildlife. Animals 2020, 10, 1500.

13. Yaribeygi, H.; Panahi, Y.; Sahraei, H.; Johnston, T.P.; Sahebkar, A. The impact of stress on body function: A review. EXCLI journal 2017, 16, 1057.

14. Denardo, D. Stress in captive reptiles. In Reptile medicine and surgery, Elsevier Inc.: 2006; pp. 119-123.

15. Norris, D.O.; Carr, J.A. Vertebrate endocrinology; Academic Press: 2020.

16. Guindre-Parker, S. Individual Variation in Glucocorticoid Plasticity: Considerations and Future Directions. Integrative and comparative biology 2020, 60, 79-88.

17. Bonino, M.F.; Azócar, D.L.M.; Schulte, J.A.; Cruz, F.B. Climate change and lizards: changing species' geographic ranges in Patagonia. Regional Environmental Change 2015, 15, 1121-1132.

18. McCallum, M.; McCallum, J.; Trauth, S. Predicted climate change may spark box turtle declines. Amphibia-Reptilia 2009, 30, 259-264.

19. Mitchell, N.J.; Allendorf, F.W.; Keall, S.N.; Daugherty, C.H.; Nelson, N.J. Demographic effects of temperature-dependent sex determination: will tuatara survive global warming? Global Change Biology 2010, 16, 60-72.

20. Storey, K.B.; Storey, J.M.; Tanino, K. Strategies of molecular adaptation to climate change: the challenges for amphibians and reptiles. Temperature Adaptation in a Changing Climate. Wallingford, UK: CABI Publishers 2011, 3, 98.

21. Hartup, B.K. Rehabilitation of native reptiles and amphibians in DuPage County, Illinois. Journal of Wildlife Diseases 1996, 32, 109-112.

Scheelings, T.F. Morbidity and Mortality of Reptiles Admitted to the Australian Wildlife Health Centre, Healesville Sanctuary, Australia, 2000-13. Journal of wildlife diseases 2015, 51, 712-718.

23. Romero, L.M.; Dickens, M.J.; Cyr, N.E. The reactive scope model-a new model integrating homeostasis, allostasis, and stress. Hormones and behavior 2009, 55, 375-389. 
24. Koenig, J.; Shine, R.; Shea, G. The ecology of an Australian reptile icon: how do blue-tongued lizards (Tiliqua scincoides) survive in suburbia? Wildlife Research 2001, 28, 214-227.

25. Fenner, A.; Hutchinson, M.; McDonald, P.; Robertson, P. Tiliqua adelaidensis. The IUCN Red List of Threatened Species 2018, e.T21902A101743579.

26. Hutchinson, M. Pogona barbata. The IUCN Red List of Threatened Species 2018, e.T170419A83493237.

27. Melville, J.; Wilson, S. Pogona vitticeps. The IUCN Red List of Threatened Species 2018, e.T83494364A83494440.

28. Kennett, R.; Roe, J.; HODGES, K.; Georges, A. Chelodina longicollis (Shaw 1794)-eastern long-necked turtle, common longnecked turtle, common snake-necked turtle. Chelonian Research Monographs 2009, 5, 031.031-031.038.

29. Georges, A.; Thomson, S. Diversity of Australasian freshwater turtles, with an annotated synonymy and keys to species. Zootaxa 2010, 2496, 1-37.

30. Shea, G. Tiliqua scincoides. The IUCN Red List of Threatened Species 2017, e.T109481538A109481555.

31. Pettigrew, M.; Bull, C.M. The impact of heavy grazing on burrow choice in the pygmy bluetongue lizard, Tiliqua adelaidensis. Wildlife Research 2011, 38, 299-306.

32. Atcheson, T. Husbandry guidelines for eastern blue tongue lizard (Tiliqua scincoides). Availabe online: http://nswfmpa.org/Husbandry\%20Manuals/Published\%20Manuals/Reptilia/Blue\%20Tongue\%20Lizard\%20(Atcheson).p df (accessed on 31 January 2021).

33. Sanderson, C.; Lloyd, R.; Craig, M.; Gaikhorst, G. Tiliqua rugosa. The IUCN Red List of Threatened Species 2017, e.T109481513A109481530.

34. Pianka, E.R.; Pianka, E.R.; Vitt, L.J. Lizards: windows to the evolution of diversity; Univ of California Press: 2003 ; Vol. 5.

35. Narayan, E.; Vanderneut, T. Physiological stress in rescued wild koalas are influenced by habitat demographics, environmental stressors, and clinical intervention. Frontiers in endocrinology 2019, 10, 18.

36. Schreck, C.B.; Tort, L.; Farrell, A.P.; Brauner, C.J. Biology of stress in fish; Academic Press: 2016.

37. Barton, B.A. Stress in fishes: a diversity of responses with particular reference to changes in circulating corticosteroids. Integrative and comparative biology 2002, 42, 517-525.

38. Asterita, M.F. The Physiology of Stress: with special reference to the neuroendocrine system; Human Sciences Press: 1985.

39. Pyke, G.H.; Szabo, J.K. What can we learn from untapped wildlife rescue databases? The masked lapwing as a case study. Pacific Conservation Biology 2018, 24, 148-156.

40. Shine, R.; Koenig, J. Snakes in the garden: an analysis of reptiles "rescued" by community-based wildlife carers. Biological Conservation 2001, 102, 271-283.

41. Charalambous, R.; Narayan, E. A 29-year retrospective analysis of koala rescues in New South Wales, Australia. Plos one 2020, 15, e0239182.

42. Hamer, A.J.; McDonnell, M.J. The response of herpetofauna to urbanization: inferring patterns of persistence from wildlife databases. Austral Ecology 2010, 35, 568-580.

43. McCormick, G.L.; Shea, K.; Langkilde, T. How do duration, frequency, and intensity of exogenous CORT elevation affect immune outcomes of stress? General and comparative endocrinology 2015, 222, 81-87.

44. Turner, G.S. Natural History Notes on the Eastern Blue-tongued Skink'Tiliqua scincoides scincoides' from the Basalt Plains around Melbourne. Victorian Naturalist, The 2010. 